IDDF2020-ABS-0220 SYSTEMATIC REVIEW WITH METAANALYSIS: ASSOCIATION OF OBESITY AND IRRITABLE BOWEL SYNDROME

${ }^{1}$ Wei Jian Neo*, ${ }^{1}$ Shu Hui Ngoh, ${ }^{2}$ Wai Mun Loo, ${ }^{2}$ Alex Yu Sen Soh, ${ }^{2}$ Jonathan Wei Jie Lee, ${ }^{3}$ Evelyn Xiu Ling Loo, ${ }^{1}$ Ing Wei Khor, ${ }^{4}$ Roger Chun Man Ho, ${ }^{2}$ Kewin Tien Ho Siah. ${ }^{1}$ Department of Medicine, Yong Loo Lin School of Medicine, National University of Singapore, Singapore; ${ }^{2}$ Division of Gastroenterology and Hepatology, University Medicine Cluster, National University Health System, Singapore; ${ }^{3}$ Department of Paediatrics, Yong Loo Lin School of Medicine, National University of Singapore, Singapore; ${ }^{4}$ Department of Psychological Medicine, Yong Loo Lin School of Medicine, National University of Singapore, Singapore

\subsection{6/gutjpl-2020-IDDF.128}

Background While many studies have found an association between irritable bowel syndrome (IBS) and obesity, many others did not. Hence, this systematic review with meta-analysis aims to evaluate the relationship between obesity and IBS, and determine whether obesity correlates differently with the various IBS endotypes.

Methods A systematic review of published literature was conducted in concordance with the PRISMA guidelines and followed by a meta-analysis of relevant studies using the random-effects model. The 34 included studies assessed obesity among non-IBS controls and IBS subjects, either as continuous or dichotomous outcomes, using body mass index (BMI) and waist circumference (WC). The standardized mean difference (SMD) and 95\% confidence interval (CI); and the odds ratio (OR) and 95\% CI were obtained for continuous and dichotomous outcomes respectively. Meta-regression was performed to identify sources of heterogeneity.

Results We found a weak association between IBS and obesity, but this was not statistically significant. For BMI, the overall SMD was +0.227 (95\% CI: -0.093 to $0.547, \mathrm{p}=0.164$ ) and OR was 1.312 (95\% CI: 0.974 to $1.767, \mathrm{p}=0.074$ ). For WC, the overall SMD was $+1.020 \mathrm{~cm}$ (95\% CI: -1.768 to $3.807, \mathrm{p}=0.473$ ). However, subgroup analysis revealed significantly higher BMI among IBS-diarrhoea (IBS-D) subjects compared to controls (SMD $=+1.940 ; 95 \%$ CI: 0.679 to $3.200, \mathrm{p}=0.003$ ) and significantly lower BMI among IBSconstipation (IBS-C) subjects compared to IBS-D subjects $(\mathrm{SMD}=-1.720 ; 95 \% \mathrm{CI}:-2.489$ to $-0.950, \mathrm{p}<0.001)$. Meta-regression identified age and gender as significant moderators contributing to heterogeneity.

Conclusions In conclusion, our systematic review with metaanalysis showed that obesity is positively associated with diarrhoea-predominant IBS, but not IBS in general. Our findings support the exploration of shared pathophysiology between IBS-D and obesity.

\section{IDDF2020-ABS-0221 ASCITIC FLUID CHOLESTEROL IS A USEFUL MARKER TO RULE OUT MALIGNANT ASCITES}

Sagar Walinjkar*, Anil Arora, Ashish Kumar, Praveen Sharma, Naresh Bansal. Institute of Liver, Gastroenterology and Pancreaticobiliary Sciences, Sir Ganga Ram Hospital, India

\subsection{6/gutjnl-2020-IDDF.129}

Background Diagnosis of malignant ascites is a clinical challenge. The gold standard method for diagnosis is with cytology; however, it has low sensitivity, is observer-dependent, and may require repeated paracentesis. Recently, some studies showed that high ascitic fluid cholesterol level could be a marker of malignant ascites and thus can differentiate between benign and malignant ascites. This study aimed to assess and evaluate the diagnostic accuracy of ascitic fluid cholesterol level in differentiating malignant from non-malignant ascites.

Methods We prospectively studied patients of ascites, who were admitted in from February 2019 to July 2019. Ascitic fluid cholesterol of all patients was sent along with cytology for malignant cells. Malignancy was proven by tissue diagnosis (peritoneal biopsy or EUS guided abdominal lymph node FNA or cytology of ascitic fluid). Patients with spontaneous bacterial peritonitis, tubercular ascites and pancreatic ascites were excluded.

Results A total of 50 patients of ascites were included in the study, and of these 11 patients had malignant ascites as proved by peritoneal biopsy or EUS guided abdominal lymph node biopsy or by ascitic fluid cytology. On taking a cut-off of $30 \mathrm{mg} / \mathrm{dL}$ of ascitic fluid cholesterol level, it was found that 9 of 11 patients $(81 \%)$ of malignant ascites had high cholesterol values; however, only 8 of 39 patients $(20.5 \%)$ of non-malignant ascites had high cholesterol values $(p<0.05)$. The sensitivity, specificity, positive predictive value, negative predictive value, and accuracy of ascitic fluid cholesterol for diagnosing malignant ascites were 81.81\%, 79.48\%, 52.94\%, $94 \%$, and $80 \%$ respectively.

Conclusions Ascitic fluid cholesterol has high negative predictive value for ruling out malignancy-related ascites and thus can be used as an excellent biochemical investigation to rule out malignant ascites.

\section{IDDF2020-ABS-0223 AN ANALYSIS OF ADVERSE EFFECTS OF THALIDOMIDE TREATMENT IN INFLAMMATORY BOWEL DISEASE WITH PERIPHERAL NEURITIS}

Xiang Peng*, Jiayin Yao, Jun Deng. Department of Gastroenterology, the Sixth Affiliated Hospital, Sun Yat-sen University, Guangdong Provincial Key Laboratory of Colorectal and Pelvic Floor Disease, China

\subsection{6/gutjnl-2020-IDDF.130}

Background Thalidomide has been gradually recognized in the treatment of IBD because of its anti-angiogenesis and immunomodulatory effect. The safety and adverse reactions of thalidomide have also attracted considerable attention. This study aims to analyze the peripheral neuropathy associated with thalidomide in IBD and to provide guidance for safe use.

Methods We analyse 176 patients with IBD. They have suffered from peripheral neuritis due to thalidomide treatment. The peripheral nerve symptoms were evaluated, the electromyography was performed, and the dose-effect relationship of neurotoxicity was analyzed.

Results 70 patients had symmetrical numbness of hand or foot, of which 57 had abnormal electromyogram. 51.8\% (55/ 106) of the patients without peripheral nerve symptoms still had abnormal electromyogram. Abnormal electromyogram usually occurred at 15 months in patients with clinical symptoms, which was significantly earlier than that in asymptomatic patients $(15.4 \pm 16.36$ vs $21.63 \pm 16.27, P=0.016)$. In patients with an abnormal electromyogram, the daily dose was 0.084 \pm 0.023 , the cumulative use time was $19.22 \pm 16.6$ months, and the cumulative dose was $65.26 \pm 90.99 \mathrm{~g}$, while in patients with a normal electromyogram, the daily dose was 0.080 $\pm 0.021 \mathrm{~g}$, the cumulative use time was $19.63 \pm 16.4$ months, 
and the cumulative dose was $51.5 \pm 124.84 \mathrm{~g}$. There was no significant difference between the two groups. Peripheral neuropathy may occur earlier in males than in females, but no significance $(36.1 \pm 96.9$ vs $72.9 \pm 118.0 \mathrm{~g}, \mathrm{P}=0.07$, and 20.0 \pm 20.1 months, $\mathrm{P}=0.18$ ).

Conclusions Whether thalidomide can lead to peripheral neuritis has a large individual difference. Male is more likely to develop peripheral neuritis than female to develop peripheral neuritis. But there is no statistical difference. Although symptomatic patients develop electromyogram damage earlier, it should be noted that there are still some patients with peripheral neuritis in asymptomatic patients. Therefore, electromyography should be monitored regularly in IBD patients with thalidomide for more than one year to avoid irreversible peripheral neuritis.

\section{IDDF2020-ABS-0224 THE RISK OF MYOCARDIAL INFARCTION IN PATIENTS WITH INFLAMMATORY BOWEL DISEASE: A META-ANALYSIS}

${ }^{1}$ Raditya Dewangga*, ${ }^{2}$ Kevin Winston, ${ }^{3}$ Lazuardi Gayu Ilhami. 'Annisa Bekasi Hospital, Indonesia; ${ }^{2}$ Faculty of Medicine Universitas Indonesia, Indonesia; ${ }^{3}$ Kartika Cibadak Hospital, Indonesia

\subsection{6/gutjnl-2020-IDDF.131}

Background Systemic inflammation of inflammatory bowel disease (IBD) may increase the risk of cardiovascular events. Yet, the risk of myocardial infarction incidence in IBD patients is still unclear. We aim to investigate the risk of myocardial infarction in patients with ulcerative colitis (UC) or Crohn's disease (CD).

Methods We systematically searched for an observational study about IBD and myocardial infarction from PubMed, EBSCO, and Scopus up to August 2020. The inclusion criteria were the diagnosis of IBD preceding the incidence of myocardial infarction and survival study providing hazard ratio. Hazard ratio (HR) was calculated with 95\% confidence interval (CI) while heterogeneity was assessed using the $\mathrm{I}^{2}$ statistics. The primary outcome was myocardial infarction incidence. Data from eligible cohort studies were pooled for effect estimates. Statistical analysis was performed using Review Manager software.

Results We selected 4 eligible cohort survival studies for analysis. The pooled hazard ratio was 1.54 (95\% CI 1.07-2.21; $\mathrm{I}^{2}$ : 84\%) for Crohn's disease and 1.29 (95\% CI 1.06-1.57; I²: 80) for Ulcerative Colitis (figure 1). High heterogeneity was attributed to the different follow-up periods and patient characteristics used in the pooled studies. The funnel plot was asymmetric, suggesting publication bias.

Conclusions There was an association between IBD and the incidence of myocardial infarction. Further study is needed to confirm the results.

\section{IDDF2020-ABS-0226 IBS AND ANIMAL EXPOSURE}

Alexa Lai*, Shirley Bong. National University Hospital, Singapore

\subsection{6/gutjph-2020-IDDF.132}

Background Irritable bowel syndrome (IBS) is a heterogenous functional disorder that is rising in worldwide prevalence with a significant healthcare burden. There has been increasing attention worldwide on the relationship between animal exposure and IBS.

Methods PubMed was used to search for all of the studies published using the keywords: 'irritable bowel syndrome' and 'pet ownership', 'giardiasis', 'blastocystis', 'aeroallergens'.

In our review, we explore the association of pet ownership and IBS and their mechanism of action.

Results Pet ownership changes the gut microbiome, which can lead to gut dysbiosis and disruption of gut immunity. Blastocystis, Bifidobacterium, Ruminococcus and Akkermansia are amongst several microbiomes affected by pet ownership and shown to be associated IBS.

Conclusions Current data delineate a clear link between animal exposure and IBS. However, more studies are warranted to substantiate these explanations and understand the relationship on an individual, societal and global level.

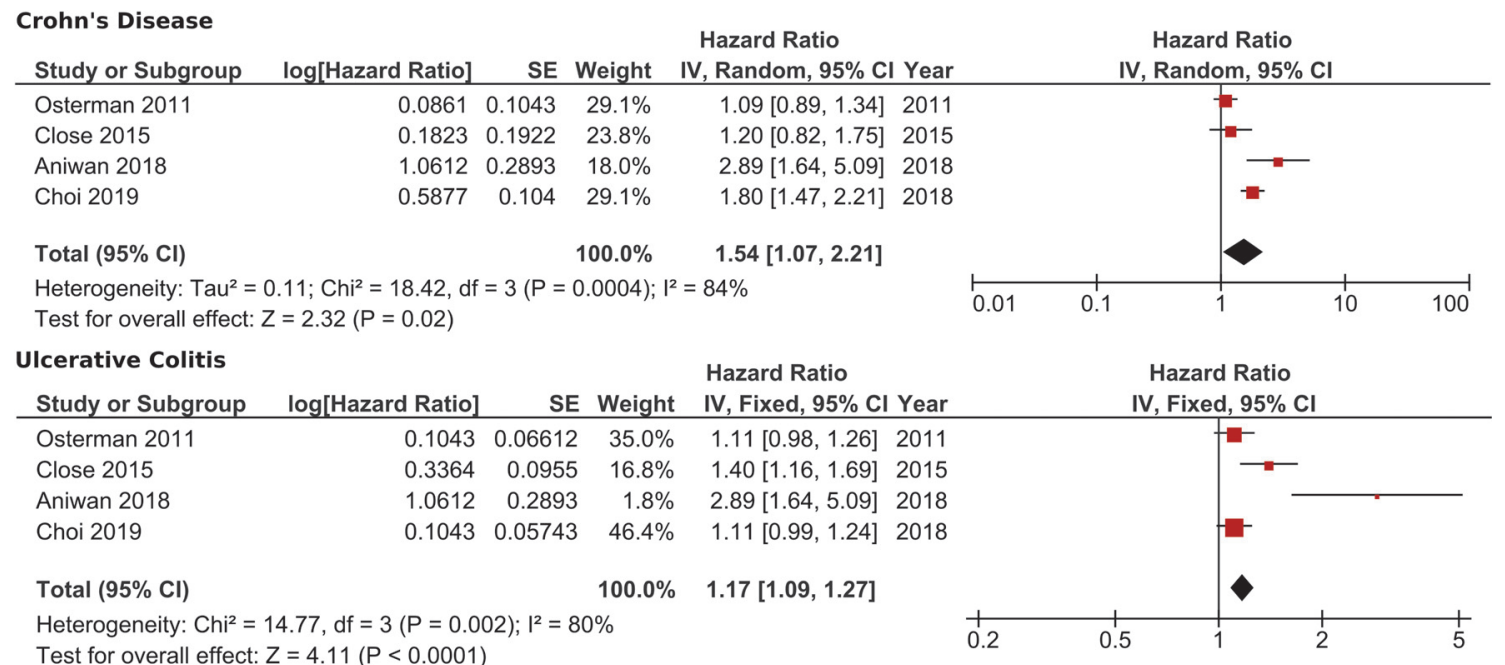

Abstract IDDF2020-ABS-0224 Figure 1 Forest plot showing the risk of myocardial infarction in Crohn's Disease and Ulcerative Colitis 